\title{
NEW ETHNOMEDICINAL INFORMATION FROM LEPCHA COMMUNITY OF DZONGU, SIKKIM
}

\author{
D.K. Pradhan ${ }^{1}$, J. Ghosh², N. Lepcha1, A. Nandi³, D. Banerjee², M. Midday², S. Naskar², D. Maity ${ }^{* 2}$ \\ Received 29 November 2021, revised 29 December 2021
}

\begin{abstract}
The Lepcha community of Sikkim is primarily confined to the Dzongu area of the state. The people of this community are mostly dependent on the forest produces for their livelihood since the ancient times and used plant resources to cure daily ailments. In the present work, extensive field surveys conducted in different corners of Dzongu to document the ethnomedicinal plants and their uses from the traditional practitioners as well as the local people. As a result of which new information on the ethnomedicinal uses of $\mathbf{4 0}$ plant species have been recorded and these are presented here for future reference. The uses of plants have been validated statistically following Use Value (UV) and Fidelity Level (FL) methods.
\end{abstract}

Key words: Dzongu, Ethnomedicine, ITK, Lepcha, Sikkim, Medicinal plants.

\section{INTRODUCTION}

Traditional healing is the age-old practice of disease curing brought about by the specific knowledge, skills, incantation and beliefs of the indigenous people spread around the globe (Castetter 1944). This treatment method is passed from ancestors to progenies to improve health, satiate agony and attain wellbeing. In most of the pharmacopeia relevance has been given to the wild plants which are indiscriminately used for the treatment of various ailments. About $85 \%$ of the medicines used by the ethnic communities globally are either plants or plant derivatives (Farnsworth 1988). In India almost $80 \%$ of the people still rely on non-allopathic treatments for their medicinal needs (Modak et al. 2015). Until now, more than 18000 flowering plant species are recorded in India (Singh and Dash 2018, Lakshminarasimhan and Arisdason 2018), out of which around 3000 species are used in different systems of medicine, i.e. $16.33 \%$ of the plants are used by the different ethnic communities to treat myriad of illness (Schippmann et al. 2002). The ancient Indian text, Charak Samhita and Sushrut Samhita, are the burning examples of the reliance on the herbal remedies for disease curing.
The dominance of these herbal remedies is mostly due to its easy availability, cheap price and almost without any side effects. However, it is worthy to mention here that proper screening of herbal medicine is very important in order to check its toxic potential which may be detrimental to the human health.

Sikkim, the $2^{\text {nd }}$ small state, located in the northern fringe of the country is an abode of unique flora and fauna (Maity 2021). The state is endowed with 4558 species of angiosperms which is nearly $26 \%$ of the total floristic diversity of the country (Maity et al. 2018, Maity 2021). Sikkim is the treasure of various ethnomedicinal plants and about 500 species are recorded having different medicinal properties (Gurung 2002, Tamsang 2004, Panda and Mishra 2012). Several candidate medicinal plants with high curative potential are already been commercially exploited by the different pharmaceutical companies (Rai and Sharma 1994a,b). In the recent past the uses of many non-conventional medicinal plants growing in this State are reported by several workers (Thapa and Chettri 1993, Maity et al. 2003, Maity et al. 2004).

The State is colonized by ethnic communities, like

${ }^{1}$ Quality Control Laboratory-HARC-Sikkim State Forest Herbarium (SSFH), Forests and Environment Department, Government of Sikkim, Gangtok-737102, India.

${ }^{2}$ Department of Botany, University of Calcutta, 35, Ballygunge Circular Road, Kolkata - 700 019, India.

${ }^{3}$ Dept. of Veterinary Parasitology, West Bengal University of Animal and Fishery Sciences, Kolkata- 700 037, India.

"Corresponding author.e-mail:debmaity@yahoo.com 


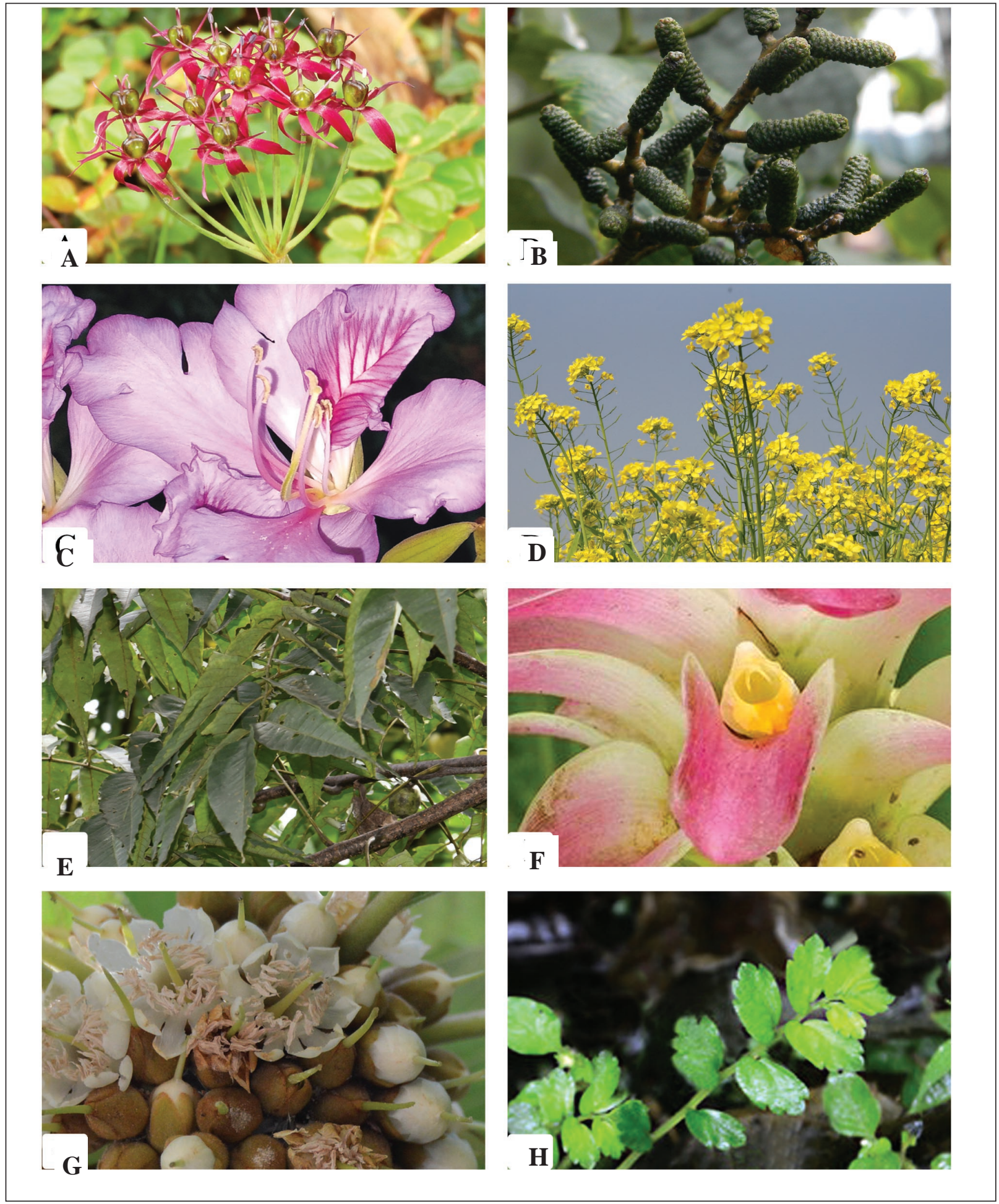

Fig. 1. A. Allium wallichii; B. Alnus nepalensis; C. Bauhinia variegata; D. Brassica nigra; E. Choerospondias axillaris; F. Curcuma caesia; G. Diploknema butyracea; H. Elatostema obtusum.

Nepalese, Bhutias, Sherpa, Rai, Limbu, Lepcha, Lachenpa, Lachungpa etc. and among them Lepcha is the original inhabitants of Sikkim (Thakur 1988, Jana and Chauhan 1999, Maity et al. 2018). The Lepcha community is primarily restricted to the Upper Dzongu and to protect their socio-religious culture this part of the State is protected under 3069/O.S dated $24^{\text {th }}$ March 1958, Home Department, Government of Sikkim. They are Mongoloid origin, quite innocent and generally prefer solitude (Thakur 1988, Maity et al. 2003). Farming is the major occupation and they commonly cultivate large cardamom, paddy, maize, millet, potato, cabbage, etc. 

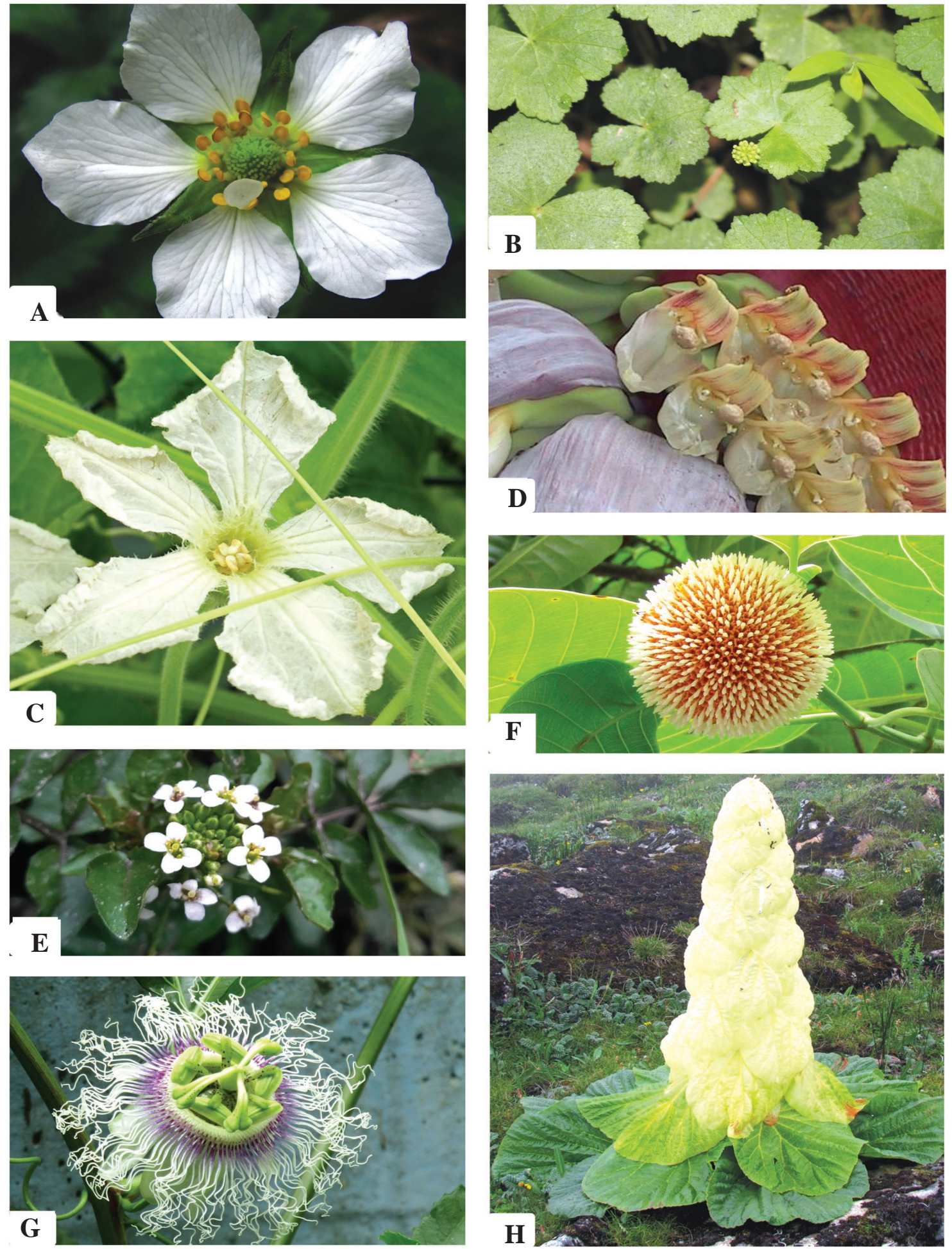

Fig. 2. A. Fragaria nubicola; B. Hydrocotyle javanica; C. Lagenaria siceraria; D. Musa balbisiana; E. Nasturtium officinale; F. Neolamarckia cadamba; G. Passiflora edulis; H. Rheum nobile.

Lepcha community holds tremendous ethnic knowledge in the utilization of forest resources. They even survive without modern facilities.

\section{MATERIALS AND METHODS}

The study area Dzongu is located at $27^{\circ} 28^{\prime}-27^{\circ} 38^{\prime} \mathrm{N}$ and $88^{\circ} 23^{\prime}-88^{\circ} 38^{\prime} \mathrm{E}$ and covers an area of about 78 sq. $\mathrm{km}$. The study area falls under the subtropical to the alpine forest covers which is predominantly explored by the 


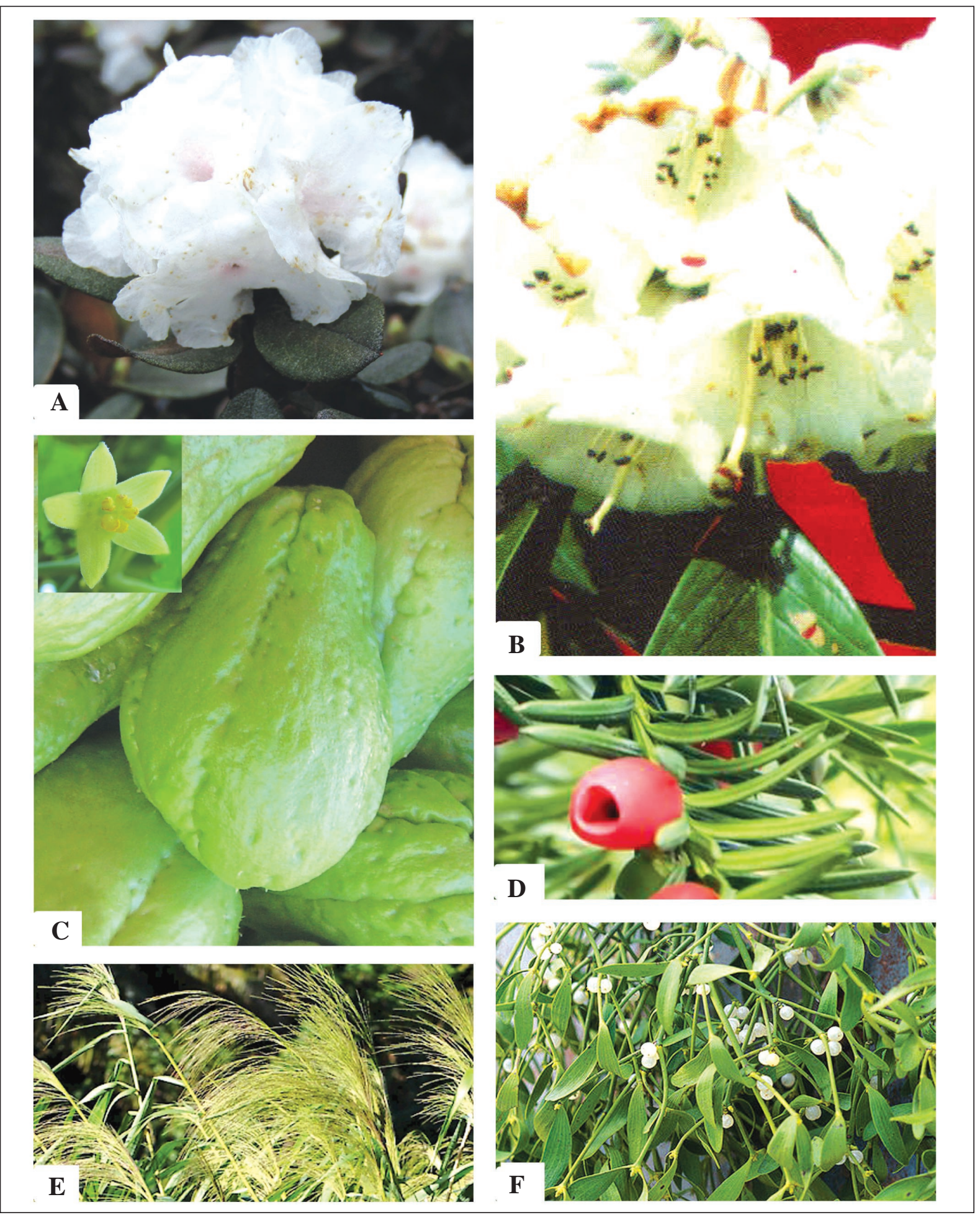

Fig. 3. A. Rhododendron anthopogon; B. Rhododendron grande; C. Sicyos edulis; D. Taxus wallichiana; E. Thysanolaena latifolia; F. Viscum album.

Lepcha community. Extensive field surveys have been conducted in the Dzongu area in the last five years. The traditional ethnomedicinal information had been gathered precisely from different medicine men as well as from the local people of this area. The uses of different plants or plant parts, their local names and parts used, mode of preparation, dosage etc. were noted carefully. The validation of information in relation to the data quantification and its efficacy in human health care was performed through statistical analyses like Use Value 
(UV) (Prance et al. 1987, Philips and Gentry 1993a,b) and Fidelity Level (FL) (Friedman et al. 1986). Representative specimens had been collected along with its color photographs for better and easy recognition of the species. Herbarium specimens were prepared following standard methodology (Ranjan 2018). All the specimens were deposited at SSFH. Extensive literature surveys were conducted to confirm the new claims. The plant species or taxa as listed, were provided with accepted scientific name, family, local name, parts used, the new use in human health care, UV, FL, preparation of medicine. The accession number of each plant species or taxa of Sikkim State Forest Herbarium (SSFH) were provided as the collected plants were deposited for future reference (Table 1).

\section{Data quantification}

Data quantification was performed following Friedman et al. (1986) for Fidelity Level (FL) and Philips and Gentry $(1993 a, b)$ for Use value (UV) to validate the reliability of ethnomedicinal information.

\section{Fidelity Level (FL):}

It were used to identify the species relating to its effectiveness and has been calculated following Friedman et al. (1986).

$$
\text { Fidelity Level }(\mathrm{FL})=\frac{\mathrm{Ip} X 100}{\mathrm{Iu}}
$$

Here, $I_{p}$ means the number of informants suggesting the use of the species and I means the total informants.

\section{Use value (UV):}

Philips and Gentry (1993a,b) had introduced this method. In this procedure the informants', knowledge of a species was calculated (Philips and Gentry 2013) by the following formula:

UVis $=\Sigma \mathrm{U}_{\text {is }} / \mathrm{n}_{\text {is }}$

Where $\mathrm{UV}_{\text {is }}=$ the use value of the species $\mathrm{S}$ mentioned by the informant $\mathrm{i} ; \Sigma \mathrm{U}_{\text {is }}=$ is the number of uses of species $\mathrm{S}$ mentioned in each event by the informant $\mathrm{i} ; \mathrm{n}_{\mathrm{is}}=$ the number of events in which the informant $i$ cited species S.

\section{RESULTS AND DISCUSSION}

The Lepcha community of Sikkim is exceptionally rich in indigenous traditional knowledge and efficiently uses forest resources in their daily lives. Under present investigation we recorded 40 medicinal plants with novel uses which had never been documented earlier. All new medicinal uses along with relevant information are appended in Table 1 .

The Lepcha community was living in the difficult areas of Dzongu. In earlier days and even today the community medicates themselves mostly by the plant materials collected directly from forest. Such information on the ethnomedicines of Lepcha community have been compiled in this article and only new claims are considered here. Colour photographs of the collected specimens helped better identification of the plants (Fig. $1,2,3)$. The age old practice of the community is of great societal value. The validation of the effectiveness and the usefulness of a species have been done through statistical analysis (Friedman et al. 1986, Prince et al. 1987, Philips and Gentry 1993a,b). Thus, the present information on new claims of medicinal uses of 38 species of seed plants and two species of pteridophytes will open a new arena in the drug discovery. Bioprospection of these species is highly recommended for the sustainable development of the state as well as the country. The information on the use of different non-conventional medicinal plants by the Lepcha community are few (Jana and Chauhan 2000, Maity et al. 2003, Tamsang 2004). The rugged terrain and the inaccessible locality often debar its inhabitants to access the modern health care facilities. Their confinement in remote areas has accelerated their reliance on the herbal medicines (Jana and Chauhan 2000). In this present investigation a total of 40 plant species (or taxa), 38 species or taxa of seed plants and two species of pteridophytes, with new medicinal uses are documented from the Lepchas of Dzongu area of Sikkim (Fig.1).

\section{ACKNOWLEDGEMENT}

Authors are thankful to the Department of Forest and Environment, Government of Sikkim for the kind support as well as the people of the Dzongu who had participated in the survey during the study. Authors thank to Mr. Norden Lepcha, Timbong, Dzongu for helping in the qualitative study to improve the content.

\section{REFERENCES}

Castetter EF (1944) The domain of ethnobiology. Amer Nat 78: $158-170$.

Farnsworth NR (1988) Screening plants for new medicines. In: Wilson EO, Peter FM (eds.), Biodiversity. Washington, DC: National Academy Press.

Friedman J, Yaniv Z, Dafni A, Palewitch D (1986) A preliminary classification of the healing potential of medicinal 
New ethnomedicinal information from Lepcha community of Dzongu, Sikkim

Table 1. Enumeration of plants with new ethnomedicinal uses by Lepcha community of Dzongu, Sikkim.

\begin{tabular}{|c|c|c|c|c|c|}
\hline Names of plants & Local names & $\begin{array}{c}\text { Parts } \\
\text { used }\end{array}$ & Uses & $\begin{array}{l}\text { UV/ } \\
\text { FL }\end{array}$ & MP \\
\hline $\begin{array}{l}\text { Allium wallichii Kunth [Alliaceae]; } \\
\text { Acc.: SSFH SK002549 }\end{array}$ & $\begin{array}{l}\text { Dungdung gay } \\
(\mathrm{N}), \text { Rumbo }(\mathrm{L})\end{array}$ & $\begin{array}{l}\text { Whole } \\
\text { plant }\end{array}$ & $\begin{array}{l}\text { As digestive, in } \\
\text { anemia }\end{array}$ & $\begin{array}{l}1.39 \\
150\end{array}$ & Aerial parts boiled in water \\
\hline $\begin{array}{llr}\text { Alnus nepalensis D. } & \text { Don } \\
\text { [Betulaceae]; Acc.: } & \text { SSFH } \\
\text { SK002565 } & & \end{array}$ & $\begin{array}{l}\text { Uttis } \quad(\mathrm{N}) \text {, } \\
\text { Sungdung kung } \\
(\mathrm{L})\end{array}$ & Bark & Antioxidant, cancer & $\begin{array}{l}1.3 / \\
70\end{array}$ & $\begin{array}{l}\text { Grind } 500 \mathrm{gm} \text {, boil in } 2 \text { litres } \\
\text { water, reduce to } 1 / 4 \text { volume, } 50 \\
\mathrm{ml} \text { thrice daily }\end{array}$ \\
\hline $\begin{array}{l}\text { Bauhinia variegate L. [Fabaceae]; } \\
\text { Acc.: SSFH SK002573 }\end{array}$ & $\begin{array}{l}\text { Koiralo }(\mathrm{N}) \\
\text { Aaraykung }(\mathrm{L})\end{array}$ & Flower & $\begin{array}{l}\text { Controlling } \\
\text { hypertension }\end{array}$ & $\begin{array}{l}1.46 \\
180\end{array}$ & $\begin{array}{l}\text { Boil } 500 \mathrm{gm} \text { in } 2 \text { litres water, } \\
\text { reduce to } 1 / 4 \text { volume, } 50 \mathrm{ml} \\
\text { thrice daily }\end{array}$ \\
\hline $\begin{array}{l}\text { Brassica nigra } \\
\text { [Brassicaceae]; } \\
\text { SK002575 }\end{array}$ & $\begin{array}{l}\text { Toori }(\mathrm{N}), \text { Kanba } \\
(\mathrm{L})\end{array}$ & $\begin{array}{l}\text { Whole } \\
\text { plant }\end{array}$ & $\begin{array}{l}\text { Weakness, menstrual } \\
\text { disorder }\end{array}$ & $\begin{array}{l}1.26 \\
170\end{array}$ & $\begin{array}{l}\text { Extract of } 500 \mathrm{gm} \text { in } 2 \text { litres } \\
\text { water, reduced to } 1 / 4 \text { volume, } \\
100 \mathrm{ml} \text { daily }\end{array}$ \\
\hline $\begin{array}{l}\text { Brassica oleracea var. botrytis L. } \\
\text { [Brassicaceae]; Acc.: SSFH } \\
\text { SK002576 }\end{array}$ & $\begin{array}{l}\text { Busty ko Phul } \\
\text { kopi }(\mathrm{N})\end{array}$ & Flower & $\begin{array}{l}\text { Asthma and kidney } \\
\text { disorder }\end{array}$ & $\begin{array}{l}1.3 / \\
65\end{array}$ & $\begin{array}{l}\text { Boiled } 500 \mathrm{gm} \text { in } 2 \text { litres } \\
\text { water, reduced to } 1 / 4 \text { volume, } \\
100 \mathrm{ml} \text { thrice daily. }\end{array}$ \\
\hline $\begin{array}{lll}\text { Calamus acanthospathus } & \text { Griff. } \\
\text { [Arecaceae]; Acc.: } & \text { SSFH } \\
\text { SK002577 } & & \end{array}$ & $\begin{array}{l}\text { Beth }(\mathrm{N}), \text { Roo } \\
(\mathrm{L})\end{array}$ & $\begin{array}{l}\text { Young } \\
\text { shoot }\end{array}$ & $\begin{array}{l}\text { Hypertension, in } \\
\text { cardiac disorder }\end{array}$ & $\begin{array}{l}1.4 / \\
65\end{array}$ & $\begin{array}{l}\text { Boiled two shoots, remove } \\
\text { water, make curry, once daily }\end{array}$ \\
\hline $\begin{array}{lrr}\text { Castanopsis } & \text { purpurella } & \text { (Miq.) } \\
\text { N.P.Balakr. } & \text { [Fagaceae]; } & \text { Acc.: } \\
\text { SSFH SK002554 } & \end{array}$ & $\begin{array}{l}\text { Jat Kuttus }(\mathrm{N}) \text {, } \\
\text { Kushyo } \\
\begin{array}{l}\text { kung (L) } \\
\text { Turol }\end{array}\end{array}$ & Fruit & $\begin{array}{ll}\text { In } & \text { nutrient } \\
\text { deficiency } & \end{array}$ & $\begin{array}{l}1.3 / \\
50\end{array}$ & $150 \mathrm{gm}$ fruits, once daily \\
\hline $\begin{array}{l}\text { Choerospondias axillaris (Roxb.) } \\
\text { B.L.Burtt \& } \quad \text { A.W.Hill } \\
\text { [Anacardiaceae]; Acc.: SSFH } \\
\text { SK002613 }\end{array}$ & $\begin{array}{l}\text { Lapsi } \quad(\mathrm{N}) \text {, } \\
\text { Slutpot }(\mathrm{L})\end{array}$ & Fruit & $\begin{array}{l}\text { In blood dysentery, } \\
\text { as appetizer }\end{array}$ & $\begin{array}{l}1.15 \\
160\end{array}$ & $\begin{array}{l}100 \mathrm{gm} \text { fruits, once or twice } \\
\text { daily }\end{array}$ \\
\hline $\begin{array}{l}\text { Cinnamomum bejolghota (Buch.- } \\
\text { Ham.) Sweet [Lauraceae]; Acc.: } \\
\text { SSFH SK002545 }\end{array}$ & $\begin{array}{l}\text { Sano Sing kawli } \\
(\mathrm{N}) \text {, Sungshoor } \\
\text { Kung (L) }\end{array}$ & $\begin{array}{l}\text { Leaf, } \\
\text { bark, } \\
\text { root }\end{array}$ & $\begin{array}{l}\text { Controlling blood } \\
\text { sugar and diarrhea }\end{array}$ & $\begin{array}{l}1.35 \\
160\end{array}$ & $\begin{array}{l}\text { Powder } 50 \mathrm{gm} \text { root and bark, } \\
5 \text { gm per day }\end{array}$ \\
\hline $\begin{array}{lll}\text { Curcuma caesia } & \text { Roxb. } \\
\text { [Zingiberaceae]; Acc.: } & \text { SSFH } \\
\text { SK002583 } & & \end{array}$ & $\begin{array}{l}\text { Kalo Haldi }(\mathrm{N}) \text {, } \\
\text { Anokbu manga } \\
(\mathrm{L})\end{array}$ & Rhizome & $\begin{array}{l}\text { Prevent food } \\
\text { poisoning and gastric } \\
\text { trouble }\end{array}$ & $\begin{array}{l}1.05 \\
170\end{array}$ & $\begin{array}{l}\text { Boil } 250 \mathrm{gm} \text { in } 500 \mathrm{ml} \text { milk, } \\
\text { reduce to } 1 / 2 \text { volume, twice } \\
\text { daily }\end{array}$ \\
\hline $\begin{array}{l}\text { Diplazium maximum (D.Don) } \\
\text { C.Chr. [Athyriaceae]; Acc.: } \text { SSFH } \\
\text { SK002585 }\end{array}$ & $\begin{array}{l}\text { Saunay ningro } \\
(\mathrm{N}) \text {, Tungtokbee } \\
(\mathrm{L})\end{array}$ & $\begin{array}{l}\text { Young } \\
\text { frond }\end{array}$ & $\begin{array}{l}\text { Controlling } \\
\text { cholesterol level }\end{array}$ & $\begin{array}{l}1.05 \\
155\end{array}$ & As curry \\
\hline $\begin{array}{l}\text { Diplazium spectabile (Wall. ex. } \\
\text { Mett.) Ching [Athyriaceae]; Acc.: } \\
\text { SSFH SK } 002586\end{array}$ & $\begin{array}{l}\text { Titay } \quad \text { Ningro } \\
(\mathrm{N}), \quad \text { Akrebu } \\
\text { Tungtokbee }(\mathrm{L})\end{array}$ & $\begin{array}{l}\text { Young } \\
\text { frond }\end{array}$ & $\begin{array}{l}\text { Maintaining } \\
\text { hypertension }\end{array}$ & $\begin{array}{l}1.16 \\
150\end{array}$ & As curry \\
\hline $\begin{array}{l}\text { Diploknema butyracea (Roxb.) H.J. } \\
\text { Lam. [Sapotaceae]; Acc.: SSFH } \\
\text { SK002589 }\end{array}$ & $\begin{array}{l}\text { Chueri }(\mathrm{N}) \text {, } \\
\text { Yealkung }(\mathrm{L})\end{array}$ & Bark & $\begin{array}{l}\text { Diabetes, stomach } \\
\text { problem, controlling } \\
\text { water leach }\end{array}$ & $\begin{array}{l}1.46 \\
155\end{array}$ & $\begin{array}{l}\text { Decoction for } 24 \text { hours, } 280 \\
\text { ml water extract as a single } \\
\text { dosage for two days }\end{array}$ \\
\hline
\end{tabular}

(Abbreviations: Acc.: Accession number; N: Nepali name, L: Lepcha name; UV: Use Value; Fl: Fidelity Level; MP: Medicine Preparation). 
Exploratory Animal and Medical Research, Vol.11, Issue 2, December, 2021

Table 1. Enumeration of plants with new ethnomedicinal uses by Lepcha community of Dzongu, Sikkim (Contd.).

\begin{tabular}{|c|c|c|c|c|c|}
\hline Names of plants & Local names & $\begin{array}{c}\text { Parts } \\
\text { used }\end{array}$ & Uses & $\begin{array}{l}\text { UV/ } \\
\text { FL }\end{array}$ & MP \\
\hline $\begin{array}{lll}\text { Elaeocarpus sikkimensis } & \text { Mast. } \\
\text { [Elaeocarpaceae]; Acc.: } & \text { SSFH } \\
\text { SK002590 } & & \end{array}$ & $\begin{array}{l}\text { Bhadrasay } \\
(\mathrm{N}) \text {, } \\
\text { Shaykyue } \\
\text { kung (L) }\end{array}$ & Fruit & $\begin{array}{l}\text { Blood } \\
\text { dysentery }\end{array}$ & $\begin{array}{l}1.6 / \\
80\end{array}$ & $\begin{array}{l}\text { Two ripen fruits daily for two } \\
\text { days }\end{array}$ \\
\hline 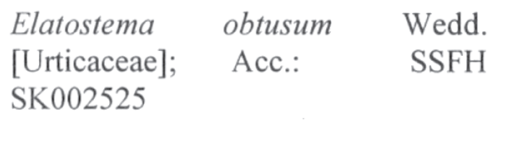 & $\begin{array}{l}\text { Goglayto } \\
(\mathrm{N}) \text {, } \\
\text { Khanshalbee } \\
\text { (L) }\end{array}$ & $\begin{array}{l}\text { Leaf and } \\
\text { stem }\end{array}$ & $\begin{array}{l}\text { Hypertension, } \\
\text { diabetes }\end{array}$ & $\begin{array}{l}1.35 \\
160\end{array}$ & As soup \\
\hline $\begin{array}{l}\text { Fragaria nubicola (Lindl. ex } \\
\text { Hook.f.) Lacaita [Rosaceae]; Acc.: } \\
\text { SSFH SK } 002591\end{array}$ & $\begin{array}{l}\text { Bhui Aiselu } \\
(\mathrm{N}), \\
\text { Zyeuepot (L) }\end{array}$ & $\begin{array}{l}\text { Aerial } \\
\text { part/ } \\
\text { Fruit }\end{array}$ & $\begin{array}{l}\text { Boosting body } \\
\text { energy }\end{array}$ & $\begin{array}{l}1.25 \\
180\end{array}$ & $\begin{array}{l}\text { Extract of } 500 \mathrm{gm} \text { in } 2 \text { litres } \\
\text { water, reduced to } 1 / 4 \text { volume, } 100 \\
\mathrm{ml} \text { twice daily/ } 4 \text { fruits, once } \\
\text { daily }\end{array}$ \\
\hline $\begin{array}{l}\text { Hydrocotyle javanica Thunb. } \\
\text { [Apiaceae]; Acc.: SSFH SK002540 }\end{array}$ & Golpatta $(\mathrm{N})$ & Leaf & Cough & $\begin{array}{l}1.63 \\
173\end{array}$ & $\begin{array}{l}\text { Extract of } 500 \mathrm{gm} \text { in } 2 \text { litres } \\
\text { water, reduced to } 1 / 4 \text { volume, } 100 \\
\text { ml once daily }\end{array}$ \\
\hline $\begin{array}{l}\text { Lagenaria siceraria (Molina) } \\
\text { Standl. [Cucurbitaceae]; Acc.: } \\
\text { SSFH SK002595 }\end{array}$ & Lauwka $(\mathrm{N})$ & Fruit & Purifying blood & $\begin{array}{l}1.15 \\
175\end{array}$ & Juice of two fruits daily \\
\hline $\begin{array}{l}\text { Litsea citrata Blume [Lauraceae]; } \\
\text { Acc.: SSFH SK002597 }\end{array}$ & Siltimbur $(\mathrm{N})$ & Fruit & Anti-infection & $\begin{array}{l}1.6 / \\
80\end{array}$ & $\begin{array}{l}\text { Grind } 10 \mathrm{gm} \text { seeds in } 1 \text { litres } \\
\text { water, reduced to } 1 / 4 \text { volume, } 10 \\
\mathrm{ml} \text { daily }\end{array}$ \\
\hline $\begin{array}{l}\text { Machilus edulis King ex } \begin{array}{r}\text { Hook. } f . \\
\text { [Lauraceae]; Acc.: }\end{array} \\
\text { SK002547 }\end{array}$ & $\begin{array}{l}\text { Phomsay }(\mathrm{N}) \\
\text { Aufompot } \\
\text { (L) }\end{array}$ & Fruit & $\begin{array}{l}\text { Controlling } \\
\text { blood sugar }\end{array}$ & $\begin{array}{l}1.16 \\
165\end{array}$ & 200 gm fruits twice daily \\
\hline $\begin{array}{l}\text { Musa balbisiana Colla [Musaceae]; } \\
\text { Acc.: SSFH SK002599 (Fig.2D) }\end{array}$ & $\begin{array}{l}\text { Bankera }(\mathrm{N}) \text {, } \\
\text { Punzok } \\
\text { kudung }(\mathrm{L})\end{array}$ & Stem & $\begin{array}{l}\text { Throat } \\
\text { infection }\end{array}$ & $\begin{array}{l}1.6 / \\
80\end{array}$ & Squeezed in water, thrice daily \\
\hline 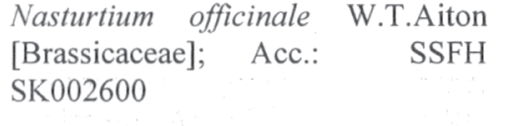 & $\begin{array}{l}\text { Simrayo }(\mathrm{N}) \text {, } \\
\text { Khaneybee } \\
(\mathrm{L})\end{array}$ & $\begin{array}{l}\text { Leaf and } \\
\text { stem }\end{array}$ & Tuberculosis & $\begin{array}{l}1.24 \\
150\end{array}$ & $\begin{array}{l}\text { Boil } 250 \mathrm{gm} \text { in } 2 \text { litres water, } \\
\text { reduced to } 1 / 4 \text { volume, } 50 \mathrm{ml} \text { once } \\
\text { daily }\end{array}$ \\
\hline $\begin{array}{l}\text { Neolamarckia cadamba (Roxb.) } \\
\text { Bosser [Rubiaceae]; Acc.: } \text { SSFH } \\
\text { SK002546 }\end{array}$ & $\operatorname{Kadam}(\mathrm{N})$ & Seed & Food poisoning & $\begin{array}{l}1.36 \\
170\end{array}$ & Decoction in water, $150 \mathrm{ml}$ \\
\hline $\begin{array}{l}\text { Neopicrorhiza } \\
\text { scrophularifflora (Pennell) } \\
\text { D.Y.Hong [Scrophulariaceae]; Acc.: } \\
\text { SSFH SK002607 }\end{array}$ & Kutki (N) & $\begin{array}{l}\text { Whole } \\
\text { plant }\end{array}$ & $\begin{array}{l}\text { Antidote } \\
\text { poison }\end{array}$ & $\begin{array}{l}1.40 \\
/ 80\end{array}$ & $\begin{array}{l}\text { Decoction of } 50 \mathrm{gm} \text { overnight, } \\
100 \mathrm{ml} \text { once daily }\end{array}$ \\
\hline $\begin{array}{lll}\text { Panax } & \text { bipinnatifidus } & \text { Seem. } \\
\text { var. angustifolius (Burkill) } & \text { J.Wen } \\
\text { [Araliaceae]; } & \text { Acc.: } & \text { SSFH } \\
\text { SK002603 } & & \end{array}$ & $\begin{array}{l}\text { Ajambhari } \\
(\mathrm{N})\end{array}$ & Rhizome & $\begin{array}{l}\text { Jaundice and } \\
\text { liver disorder }\end{array}$ & $\begin{array}{l}1.45 \\
160\end{array}$ & $\begin{array}{l}\text { Grind and boiled in } 500 \mathrm{ml} \text { milk, } \\
\text { reduced to } 3 / 4 \text { volume, } 250 \mathrm{ml} \\
\text { once daily }\end{array}$ \\
\hline $\begin{array}{l}\text { Passiflora } \quad \text { edulis } \\
\text { [Passifloraceae]; } \\
\text { SK002550 }\end{array}$ & $\begin{array}{l}\text { Garrendal } \\
\text { (N), Ranipot } \\
\text { (L) }\end{array}$ & $\begin{array}{l}\text { Leaf and } \\
\text { fruit }\end{array}$ & $\begin{array}{l}\text { Boosting } \\
\text { immunity, } \\
\text { support heart } \\
\text { health }\end{array}$ & $\begin{array}{l}1.46 \\
160\end{array}$ & $\begin{array}{l}\text { Boil } 200 \mathrm{gm} \text { in } 2 \text { litres water, } \\
\text { reduce to } 1 / 2 \text { volume, } 100 \mathrm{ml} \\
\text { thrice daily }\end{array}$ \\
\hline
\end{tabular}

(Abbreviations: Acc.: Accession number; N: Nepali name, L: Lepcha name; UV: Use Value; Fl: Fidelity Level; MP: Medicine Preparation). 
Table 1. Enumeration of plants with new ethnomedicinal uses by Lepcha community of Dzongu, Sikkim (Contd.).

\begin{tabular}{|c|c|c|c|c|c|}
\hline Names of plants & Local names & $\begin{array}{l}\text { Parts } \\
\text { used }\end{array}$ & Uses & $\begin{array}{l}\mathbf{U V} / \\
\text { FL }\end{array}$ & MP \\
\hline $\begin{array}{l}\text { Phoebe attenuata (Nees) Nees } \\
\text { [Lauraceae]; Acc.: SSFH } \\
\text { SK002535 }\end{array}$ & $\begin{array}{ll}\text { Angeri } & (\mathrm{N}), \\
\text { Kuntu }(\mathrm{L}) & \end{array}$ & Leaf & $\begin{array}{l}\text { Control pest, } \\
\text { parasite and plant } \\
\text { disease }\end{array}$ & $\begin{array}{l}1.30 \\
170\end{array}$ & $\begin{array}{l}500 \mathrm{gm} \text { in } 2 \text { litres water, reduced to } \\
1 / 4 \text { volume, once daily externally }\end{array}$ \\
\hline $\begin{array}{l}\text { Piper hamiltonii C. } \quad \text { DC. } \\
\text { [Piperaceae]; SSFH SK002553 }\end{array}$ & $\begin{array}{l}\text { Jungali Paan }(\mathrm{N}) \text {, } \\
\text { Teenrek (L) }\end{array}$ & Leaf & $\begin{array}{l}\text { Sore throat, } \\
\text { respiratory } \\
\text { disorders }\end{array}$ & $\begin{array}{l}1.43 \\
160\end{array}$ & $\begin{array}{l}\text { Intake of one or two leaves thrice } \\
\text { daily }\end{array}$ \\
\hline $\begin{array}{l}\text { Rheum nobile Hook. f. \& } \\
\text { Thomson [Polygonaceae]; Acc.: } \\
\text { SSFH SK } 002608\end{array}$ & $\begin{array}{l}\text { Paddamchal }(\mathrm{N}) \\
\text { Sanakundung }(\mathrm{L})\end{array}$ & $\begin{array}{l}\text { Stem, } \\
\text { rhizome }\end{array}$ & $\begin{array}{l}\text { Fever, cough, } \\
\text { indigestion }\end{array}$ & $\begin{array}{l}1.36 \\
170\end{array}$ & $\begin{array}{l}\text { Intake of } 1 / 2 \text { inches of part twice } \\
\text { daily }\end{array}$ \\
\hline $\begin{array}{l}\text { Rhododendron anthopogon } \\
\text { D.Don [Ericaceae]; Acc.: } \\
\text { SSFH SK002609 }\end{array}$ & $\begin{array}{l}\text { Sunpati }(\mathrm{N}) \\
\text { Bhalu chalu }(\mathrm{L})\end{array}$ & $\begin{array}{l}\text { Whole } \\
\text { plant }\end{array}$ & Bones & $\begin{array}{l}1.63 \\
180\end{array}$ & Intake of 3 to 4 leaves once daily \\
\hline 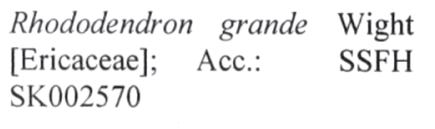 & $\begin{array}{l}\text { Guras }(\mathrm{N}) \text {; Etok- } \\
\text { Kung-Reep }(\mathrm{L})\end{array}$ & Flower & $\begin{array}{l}\text { Throat infections, } \\
\text { bronchitis, nerve } \\
\text { stimulant }\end{array}$ & $\begin{array}{l}1.6 / \\
80\end{array}$ & Traditional liquor preparation \\
\hline $\begin{array}{lrl}\text { Rubus acuminatus } & \text { Smith } \\
\text { [Rosaceae]; Acc.: } & \text { SSFH } \\
\text { SK002559 } & & \end{array}$ & $\begin{array}{l}\text { Aiselu } \\
\text { Kushyem } \\
\text { (Lep) }\end{array}$ & $\begin{array}{l}\text { Fruit, } \\
\text { shoot, } \\
\text { root }\end{array}$ & $\begin{array}{l}\text { Fever, colic, cough, } \\
\text { sore throat }\end{array}$ & $\begin{array}{l}1.36 \\
170\end{array}$ & $\begin{array}{l}\text { Extract of } 100 \mathrm{gm} \text { in } 2 \text { litres water, } \\
\text { reduced to } 1 / 4 \text { volume, } 50 \mathrm{ml} \text { daily }\end{array}$ \\
\hline $\begin{array}{l}\text { Sicyos edulis } \\
\text { [Cucurbitaceae]; Acc.: }\end{array}$ & $\begin{array}{l}\text { Iskus } \quad(\mathrm{N}) \text {, } \\
\text { Beepot/ } \\
\text { kathekpot }(\mathrm{L})\end{array}$ & Fruit & $\begin{array}{l}\text { Cancer, heart } \\
\text { problems, } \\
\text { immunity boosting }\end{array}$ & $\begin{array}{l}1.60 \\
180\end{array}$ & $\begin{array}{l}500 \mathrm{gm} \text { boiled to extract juice, } 100 \\
\text { ml twice daily }\end{array}$ \\
\hline $\begin{array}{l}\text { Smilax aspericaulis } \text { Wall. ex A. } \\
\text { DC. [Smilacaceae]; Acc.: } \\
\text { SSFH SK002611 }\end{array}$ & $\begin{array}{l}\text { Kukur Dhaney } \\
(\mathrm{N}), \text { Palamzyue } \\
(\mathrm{L})\end{array}$ & $\begin{array}{l}\text { Young } \\
\text { shoot }\end{array}$ & $\begin{array}{l}\text { High pressure, } \\
\text { diabetes }\end{array}$ & $\begin{array}{l}1.16 \\
160\end{array}$ & 250 gm used as curry once daily \\
\hline $\begin{array}{l}\text { Stephania glandulifera Miers } \\
\text { [Menispermaceae]; Acc.: SSFH } \\
\text { SK002569 }\end{array}$ & Tamarkay $(\mathrm{N})$ & $\begin{array}{l}\text { Young } \\
\text { leaf, } \\
\text { rhizome }\end{array}$ & $\begin{array}{l}\text { Diabetes, as fodder } \\
\text { tô animal }\end{array}$ & $\begin{array}{l}0.98 \\
177\end{array}$ & $\begin{array}{l}\text { Boil } 100 \mathrm{gm} \text { to extract juice, } 30 \mathrm{ml} \\
\text { twice daily }\end{array}$ \\
\hline 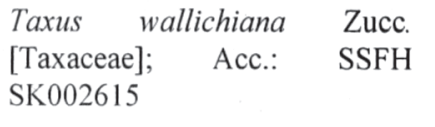 & $\begin{array}{l}\text { Lad Salla }(\mathrm{N}) \text {, } \\
\text { Pungshing }(\mathrm{L})\end{array}$ & Leaf & Dog bites & $\begin{array}{l}1.32 \\
180\end{array}$ & $\begin{array}{l}100 \mathrm{gm} \text { boiled to extract juice, } 50 \mathrm{ml} \\
\text { twice daily }\end{array}$ \\
\hline $\begin{array}{l}\text { Thysanolaena latifolia (Roxb. } \\
\text { ex Hornem.) Honda [Poaceae]; } \\
\text { Acc.: SSFH SK002558 }\end{array}$ & $\begin{array}{ll}\text { Amliso } & (\mathrm{N}) \\
\text { Pushyur (L) }\end{array}$ & Root & Mumps, abscesses & $\begin{array}{l}1.45 \\
160\end{array}$ & $\begin{array}{l}\text { Extract of } 10 \mathrm{gm} \text { in } 150 \mathrm{ml} \text { water } \\
\text { reduced to } 1 / 4 \text { volume, once daily }\end{array}$ \\
\hline $\begin{array}{l}\text { Tupistra nutans } \text { Wall. ex Lindl. } \\
\text { [Asparagaceae]; Acc.: SSFH } \\
\text { SK002614 }\end{array}$ & $\begin{array}{l}\text { Nakima } \\
\text { Perfek }(\mathrm{L})\end{array}$ & Flower & Hypertension & $\begin{array}{l}1.43 \\
144\end{array}$ & $\begin{array}{l}100 \mathrm{gm} \text { boiled to extract juice, } 50 \\
\mathrm{ml} \text { twice daily }\end{array}$ \\
\hline $\begin{array}{l}\text { Viscum album } \\
\text { [Loranthaceae]; } \quad \text { Acc.: } \\
\text { SK002618 }\end{array}$ & $\operatorname{Harchur}(\mathrm{N})$ & $\begin{array}{l}\text { Whole } \\
\text { plant }\end{array}$ & $\begin{array}{l}\text { Body ache, } \\
\text { fracture, } \\
\text { dislocation, gout, } \\
\text { gastric problems }\end{array}$ & $\begin{array}{l}1.4 / \\
80\end{array}$ & $\begin{array}{l}\text { Grind } 25 \mathrm{gm} \text {, powder taken twice } \\
\text { daily }\end{array}$ \\
\hline $\begin{array}{l}\text { Zanthoxylum rhetsa (Roxb.) } \\
\text { DC. [Rutaceae]; Acc.: SSFH } \\
\text { SK002534 }\end{array}$ & $\begin{array}{l}\text { Bokay Timbur } \\
(\mathrm{N}), \text { Yepmu }(\mathrm{L})\end{array}$ & Fruit & Fever, body pain & $\begin{array}{l}1.55 \\
180\end{array}$ & $\begin{array}{l}100 \mathrm{gm} \text { powder in } 2 \text { litres water, } \\
\text { reduced to } 1 / 4 \text { volume, } 20 \mathrm{ml} \text { once } \\
\text { daily }\end{array}$ \\
\hline
\end{tabular}

(Abbreviations: Acc.: Accession number; N: Nepali name, L: Lepcha name; UV: Use Value; Fl: Fidelity Level; MP: Medicine Preparation). 
plants, based on a rational analysis of an ethnopharmacological field survey among Bedouins in the Negev Desert, Israel J Ethnopharmacol 16: 275-287.

Gurung B (2002) The medicinal plants of the Sikkim Himalaya. Jasmine Bejoy Gurung, Maples, Chakung, West Sikkim.

Jana SK, Chauhan AS (1999) Studies on the Lepcha medicobotany of Dzongu, Sikkim Himalaya. Himalayan Paryavaran 6: 121-126.

Jana SK, Chauhan AS (2000) Ethnobotanical studies on Lepchas of Dzongu, North Sikkim. Ann For 8(1): 131-144.

Lakshminarasimhan P, Arisdason W (2018) Diversity of algae, fungi, lichens and non-flowering and flowering plants of India: an overview. In: Chourasia HK, Mishra DP (eds.), Plant systematics and biotechnology: challenges and opportunities. Today and Tomorrow's Printers and Publishers, New Delhi 110 002, India. 43-61.

Maity D (2021) Flora of Sikkim - A synoptic view. In: Singh LJ, Ranjan V (eds.), New vistas in Indian flora. Vol. II. Bishen Singh Mahendra Pal Singh. 469-496.

Maity D, Chauhan AS, Maiti GG (2003) Ethnomedicinal notes on some unexploited medicinal plants used by Lecpcha and Nepalese communities of North Sikkim. J Econ Taxon Bot 325-332.

Maity D, Maiti GG, Chauhan AS (2018) Flora of Kanchenjunga biosphere reserve, Sikkim. Botanical Survey of India, Kolkata.

Maity D, Pradhan N, Chauhan AS (2004) Folk uses of some medicinal plants from North Sikkim. Indian J Trad Knowl 3(1): 66-71.

Maity D, Pradhan N, Maiti GG (2003) Traditional uses of some medicinal plants of Sikkim Himalaya. ECOBIOS 2(1\&2): 46-56.

Modak BK, Gorai P, Dhan R, Mukherjee A, Dey A (2015) Tradition in treating taboo: Folkloric medicinal wisdom of the aboriginals of Purulia district, West Bengal India against sexual, gynaecological and related disorders. J Ethnopharma 169: 370386.

Panda AK, Mishra S (2012) Plant biodiversity of Sikkim and Ayurvedic health care system. In: Tamang P, Srivastava
AK, Lepcha SR (eds.), Sikkim biodiversity, significance and sustainability. Sikkim State Council of Science \& Technology, Department of Science \& Technology \& Climate Change, Govt. of Sikkim, Gangtok. 33-37.

Philips O, Gentry AH (1993a) The useful plants of Tambopata, Peru: I. Statistical hypotheses tests with a new quantitative technique. Econ Bot 47(1): 15-32.

Philips O, Gentry AH (1993b) the useful plants of Tambopata, Peru: II. Additional hypothesis testing in quantitative ethnobotany. Econ Bot 47(1): 33-43.

Prance GT, Balee W, Boom BM, Carneiro RL (1987) Quantitative ethno botany and the case for conservation in Amazonia. Conserv Biol 1(4): 296-310.

Rai LK, Sharma E (1994a) Medicinal plants of Sikkim Himalaya, status, uses and potential. Himavikas occasional publication no. 5. G. B. Pant institute of Himalayan environment and development. Bishen Singh Mahendra Pal Singh, Dehradun.

Rai LK, Sharma E (1994b) Status of medicinal plants from the Sikkim Hills. Sikkim Sci Soc Newsletter 7(2): 4-8.

Ranjan V (2018) Herbaria: a systematic repository of plant specimens for research. In: Maity D (ed.), Taxonomy: Theory and practice [Procedings of the first international workshop under Taxonomy training centre, AICOPTAX, MoEF \& CC, Govt. of India]. Ruby Das, Serampore Hooghly. 96-118.

Schippmann U, Leaman DJ, Cunningham AB (2002) Impact of cultivation and gathering of medicinal plants on biodiversity: Global trends and issues. (Case study No. 7). In : Biodiversity and the Ecosystem Approach in Agriculture, Foresty and Fisheries, $9^{\text {th }}$ session of the commission on 'Genetic Resources for Food and Agriculture' Rome, FAO Decument Repository of United Nations.

Singh P, Dash SS (2018) Plant diversity in Indian Himalayan regions: An overview. In: Das AP, Bera S (eds.), Plant diversity in the Himalaya hotspot region. Bishen Singh Mahendra Pal Singh, Dehra Dun. 1-46.

Tamsang KP (2004) Glossary of Lepcha Medicinal Plants. Mani Printing Press, Kalimpong, India.

Thakur RN (1988) Himalayan Lepchas. Archives Publication, New Delhi.

Thapa KK, Chettri R (1993) Ethno-botanical survey of Darjeeling-Sikkim hills. Bull Res Cell 1(2): 1-7.

Cite this article as: Pradhan DK, Ghosh J, Lepcha N, Nandi A, Banerjee D, Midday M, Naskar S, Maity D (2021) New ethnomedicinal information from Lepcha community of Dzongu, Sikkim. ExplorAnim Med Res 11(2): 179187. DOI : 10.52635/eamr/11.2.179-187. 